\title{
La quimera de una educación sin valores
}

\section{The delusion of an education without values}

\author{
Cristián David Expósito ${ }^{1}$ \\ Centro de Investigaciones Cuyo "Dr. Abelardo Pithod" - CONICET, \\ Mendoza, Argentina ${ }^{1}$
}

(iD) ORCID: http://orcid.org/0000-0002-5314-9752 ${ }^{1}$

Recibido: 02 de enero de 2020

Aceptado: 15 de julio de 2020

\begin{abstract}
Resumen
La escuela es el espacio real y concreto donde se cristalizan aquellos valores trazados en el diseño curricular propio de cada sistema educativo. Es aquí donde debe producirse también una interacción reflexiva que suscite el cuestionamiento de las costumbres culturales vigentes y de aquellos valores sociales y personales más convenientes para promover el bien de la comunidad. Esto conlleva la responsabilidad de plantear adecuaciones para mejorar la convivencia en sociedad sin comprometer la propia raíz cultural. El delicado equilibro entre estas variables devendrá en armonía; de lo contrario, obtendremos una crisis que generará posturas antagónicas y en el lugar de la paz solo encontraremos una brecha social. En este artículo abordaremos la profunda relación existente entre la tríada "educación, valores e ideología"; ya que toda situación escolar, sea cual fuere la postura ideológica asumida, va a estar impregnada de determinados valores que impactan en la educación de las personas por el modo en que son concebidos y plasmados en el currículum.
\end{abstract}

Keywords: Axiología - valores - educación - psicología social - pedagogía 


\begin{abstract}
The school is the real and concrete space where those values traced in the curricular design of each educational system crystallize. It is here that there should also be a reflexive interaction that questions the citizen about the current cultural customs and those social and personal values more convenient to promote the welfare of the community. This entails the responsibility of raising adjustments to improve coexistence in society without compromising one's cultural roots. The delicate balance between these variables will produce harmony; otherwise, we will obtain a crisis that will generate antagonistic positions and in the place of peace we will only find a social gap. In this article we will address the deep relationship between the triad "education, values and ideology"; since every school situation, whatever the ideological position assumed, will be impregnated with certain values that impact on the education of people by the way they are conceived and incorporated into the curriculum.
\end{abstract}

Keywords: Axiology - values - education - social psychology - pedagogy

\title{
Introducción
}

Indefectiblemente hablar de educación es hablar de valores. La educación es el baluarte por excelencia del ser humano en todos los tiempos y en todas las civilizaciones. Es el complejo instrumento social para la transmisión del saber, la cultura y los valores, propiamente dichos, de una sociedad (Berríos \& Buxarrais. 2013; y Gómez, 2017). En palabras de Enrique Gervilla (2000):

Todo acto educativo conlleva siempre una relación, explícita o implícita, al valor, por cuanto la educación en su misma esencia y fundamento es valiosa. De aquí que sea reiterativa la expresión «educar en valores», ya que no hay otra posibilidad de educar más que en valores. (p. 39)

Es una quimera plantear que se puede educar sin la presencia de los valores ya que estaríamos suprimiendo en propio acto educativo. "Bajo la óptica de la hermenéutica crítica, la educación es valiosa por sí misma porque en virtud de ella (la educación críticamente comprendida) el ser humano puede desarrollarse plenamente como tal" (Gracia \& González, 2018, p. 90). El hecho educativo posee un fundamento axiológico en su objetivo, proceso y resultado. A lo largo de la historia de la humanidad, diferentes definiciones de educación, provenientes de diversas culturas en toda la extensión de nuestro planeta, han dejado constancia de ello. En todas existe el planteo explícito o implícito de una mejora para el hombre en algún sentido (García, Ruiz, \& García, 2016).

La UNESCO (1974), uno de los organismos reconocido internacionalmente por la mayoría de los países del mundo, señala en la Recomendación sobre la educación para la comprensión, la 
cooperación y la paz internacionales y la educación relativa a los derechos humanos y las libertades fundamentales, que por educación se debe entender:

...el proceso global de la sociedad, a través del cual las personas y los grupos sociales aprenden a desarrollar conscientemente en el interior de la comunidad nacional e internacional y en beneficio de ellas, la totalidad de sus capacidades, actitudes, aptitudes y conocimientos. Este proceso suele limitarse, algunas veces, a una actividad determinada. (p. 154)

Por lo tanto, entendemos que la educación prepara para la convivencia social porque nos permite el reconocimiento del otro. A tal punto esto es así que Santos (2016) sostiene que: “... hay que admitir que los valores son el termómetro humano para evaluar la entropía de la raza que si no se reinventa se autodestruirá por la dinámica interna de sus propios conflictos” (p. 128). Y es aquí donde la educación manifiesta su contenido axiológico que promueve la culturalización del ciudadano en el ejercicio de los valores, los cuales son cognoscibles, enseñables y realizables (Touriñán-López, 2005).

En esta comunicación trataremos de explicar por qué es imposible separar la educación de los valores y cómo es la profunda relación que existe en la naturaleza de ambas realidades, sin perder de vista el papel relevante que desempeña la ideología dominante en el diseño curricular educativo y axiológico. Toda situación escolar, sea cual fuere la modalidad, nivel, docente, tipos de gestión o institución, va a estar regulada por una estructura de valores, la cual se manifestará de manera ejecutiva en el currículum. Las diferentes teorías axiológicas impregnan de una manera determinada el modelo curricular y repercuten en sus estudiantes de manera directa e indirecta, dando verdadero sentido al estudio de los valores en el ámbito educativo.

\section{Aproximación a la idea del valor}

La palabra "valor" es un término cargado de significado ideológico. Ha sido definido por expertos a lo largo de los siglos de muchas maneras. Cada filósofo o académico que ha estudiado esta materia termina aportando una definición propia del vocablo. No es la intención de este punto realizar un análisis histórico del concepto, sino presentar una aproximación a la idea del valor. Desde una perspectiva más bien integradora, Gervilla (2000) define al valor como “...una cualidad real o ideal, deseada o deseable por su bondad utópica, cuya fuerza estimativa orienta nuestra vida 
humana" (p. 54). En una misma línea, pero enfocada en el propio comportamiento del hombre, sostiene Hernández-Romero (2020) que el valor es "una guía o patrón de comportamiento que orientan los modos particulares de ser y vivir del individuo. Además, forman el criterio de juicio, preferencia y elección que conducen a una determinada acción" (p. 131). La amplitud y complejidad de este concepto se debe a la intimidad que guarda con el hombre y todo su entorno. Su ámbito de estudio es el de la reflexión filosófica, no obstante...

el concepto de valor, más que cualquier otro, es el concepto capital en todas las ciencias sociales. Es la principal variable dependiente en el estudio de la cultura, de la sociedad y de la personalidad, y la principal variable independiente en el estudio de las actitudes y de la conducta social" (Rokeach, 1973 cit. en García Hoz, 1993, p. 154).

En este sentido, el valor es el elemento central, "la piedra filosofal", que le permite al hombre aprender, educarse y trascender como tal. Por este motivo, consideramos que los valores son cualidades estructurales que se presentan como modos de ser (en acto y potencia) de la realidad. Es el hombre un ser capaz de crear valores, de convertir lo posible en realidad proyectando objetivos valiosos para su vida, de tal manera que configure su propio mundo en potencia y se realice como tal.

\section{El valor como componente esencial de la educación}

Toda acción de educar tiende a cristalizar una determinada estructura axiológica, es por esta razón que los valores son necesarios para interpretar el fenómeno educativo. Por su naturaleza, la educación se dirige a aquello que tiene valor y esto es sumamente claro para aquel que educa, lo cual evidencia que es un valor en sí misma y lo axiológico es inherente al propio concepto de educación (Ferrández \& Sarramona, 1997; Brezinka, 2007).

Tal como se plantea en la introducción, la educación implica la idea de perfeccionamiento de algún aspecto del hombre; en general refiere a mejoras de las facultades humanas llevando a la persona desde un estado real, "lo que es" a un estado ideal, un "deber ser" deseable. Este planteo atraviesa los siglos y aun desafía a la humanidad. En esto, Martínez (2017) sostiene que:

Educar significa potenciar al máximo las capacidades personales y académicas de todos los implicados en el proceso educativo para lograr un mayor rendimiento, desarrollar estrategias para el éxito personal y social y favorecer las competencias para el cambio y la adaptación que garanticen el éxito de una persona en un contexto social como el actual (p. 14). 
La educación es un proceso que tiende a un fin determinado que se considera valioso en sí mismo. A su vez, el valor es un fin deseable al que cada hombre tiende. En consecuencia, el valor en cuanto tal terminaría orientando todo el proceso educativo. A lo largo de los años, varios autores, al referirse al concepto de educación, lo hacen desde la axiología. Para citar algunos ejemplos traemos a colación a dos de los referente más relevantes de la filosofía occidental: Aristóteles (1993[1985]) en la Ética a Nicómaco sostiene que es necesario ordenar los sentimientos de placer y dolor a la virtud moral, que en esto consiste la buena educación; y también Immanuel Kant (2009) que un año antes de su muerte hace un planteo en su obra Sobre Pedagogía donde esboza que "(...) detrás de la educación está escondido el gran misterio de la perfección de la naturaleza humana." (p. 32).

Sería agobiante realizar un análisis crítico del sinnúmero de definiciones que pululan sobre educación; sin embargo, todas ellas abordan el concepto del valor de manera directa o indirecta. Esto es indefectible porque el valor es un componente esencial de la educación.

Por ello, los valores se conciben como parte de la cultura y como componentes de la estructura de la personalidad, con un nivel de complejidad dado por su carácter totalizador y generalizador y en la cual se produce la unidad de lo cognitivo y lo afectivo. (Ramírez, Hernández y Viamontes, 2018, p. 196)

Es el hombre, como miembro de una comunidad, el destinatario final de un modelo axiológico determinado; ya que el concepto de educación en nuestros días se sustenta en el principio de formar a una persona apta para vivir en armonía social.

La educación es una prioridad nacional y se constituye en política de Estado para construir una sociedad justa, reafirmar la soberanía e identidad nacional, profundizar el ejercicio de la ciudadanía democrática, respetar los derechos humanos y libertades fundamentales y fortalecer el desarrollo económico-social de la Nación. (Ley N 26206, 2006, art. 3)

Por consiguiente, cada sistema educativo plantea un arquetipo de hombre como fin deseable, busca formar el mejor modelo posible de persona y, en consecuencia, un prototipo óptimo de sociedad. Es decir que incursiona tanto en la esfera individual como en aquellos atributos sociales del hombre desde el concepto de educación integral (Ley No 26206, 2006, art. 4). Sanvisens (1984) enfatiza que, una de las características propias de la educación, es la de orientarse o dirigirse hacia ciertos patrones o valores que ordenan su acción informativa y directiva. Estos 
patrones forman parte del sistema educativo de un modo implícito o explícito, sean conscientes o no.

(...) aunque en la educación, en sentido estricto y riguroso, los valores como fines u objetivos deben tender a ser plenamente conscientes, admitidos racional y voluntariamente e, incluso, sometidos a crítica suficiente acerca de su validez, importancia y grado de viabilidad y aprovechamiento. (...) dichos valores presuponen (...) una ideología, una concepción del hombre, del mundo y de la vida, y que inciden tanto en el campo sociocultural como en el antropológico y ético. (Sanvisens, 1984, pp. 18-19)

Por ende, cada ideología posee diferentes escalas de valores debido a que parten de disímiles modelos antropológicos, en consecuencia, las metas educativas probablemente difieran entre sí. Ahora bien, sea cual fuere la ideología dominante, el acto educativo tiene que ser una tarea perfectiva y optimizadora de la naturaleza humana; de lo contrario, no podríamos denominar este hecho como "educación".

\section{Relación entre educación, valores e ideología}

En los sistemas educativos de diferentes países hay referencias explícitas a los valores. Los modelos axiológicos predominantes son los que permiten que prevalezcan unos valores sobre otros; sin embargo, no se puede negar su presencia como una constante universal en los diseños curriculares de todos los países. Ahora bien, la situación es que, por lo general, toda ideología admite un doble sentido que promueve el hecho de que no siempre lo que se dice es lo que realmente se transmite (Chancusi y Peralvo, 2016). Bajo este concepto, Sauter y Rodríguez (2005) presentan una reflexión de Puelles (1986) que nos afirma que:

(...) la ideología se emplea en una doble acepción, hoy comúnmente admitida: de un lado, como concepción del mundo propia de un determinado grupo histórico concreto; del otro, como deformación de la realidad, como falsa representación que emana de un grupo social; enmascara una situación de intereses ligada a una estructura social determinada (Puelles-Benítez, 1986; cit. en Sauter y Rodríguez, 2005, p. 106).

La educación promueve aquellos valores que están aceptados por la ideología dominante. Sin embargo, la ideología imperante no garantiza la satisfacción de las necesidades de todas las personas ya que, a su vez, también existen grupos particulares que bregan por anteponer sus intereses reinterpretando los principios de la ideología. Sostiene Casillas (2019) que: "En ese sentido, se hace una apreciación crítica de reconocer los valores, la ideología y la práctica política que se esconde tras la práctica del currículum” (p. 6). Ante esta situación Torres (2002) plantea que: 
Ocultar los propios valores (...) cuando realmente se viven como algo valioso para uno mismo y para los demás, además de imposible sería antihumano. Manipular la educación para imponer unos valores determinados, además de antihumano sería deshumanizador. Probablemente se encuentre cerca de este entramado de ideas alguno de los ejes del debatido pluralismo ideológico en la escuela (p. 32).

Ahora bien, la neutralidad axiológica es imposible debido a que, en educación, es necesario partir de una cosmovisión, lo que implica asumir una ideología que brinde el marco que permita posicionarse y dirigir la acción; además, es necesaria para brindarle un sentido crítico que posibilite interpretar y juzgar valores. Este sentido crítico es el "antídoto eficaz de (contra) toda manipulación" (Cantero y García 2012, p. 264).

Por ello, es necesario realizar distinciones entre educación como realidad y educación como valor. El estudio de las características que posee el diseño curricular, desde lo ideológico, en una determinada comunidad implica la incumbencia de la sociología. Pero, si se trata de la educación acaecida en una determinada etapa de la historia de dicha población, es un estudio que compete a la historia como ciencia social. Sin embargo, en ambos casos el objeto de la investigación es formar un juicio sobre el modelo axiológico de la ideología predominante. Saber qué hay y qué ha habido en materia de influencia ideológica en educación en ese lugar en un determinado momento de la historia (Avilés, 2018). Se pretende comprender qué educación se dio y qué educación se da, sin embargo, cuando se plantea qué educación se dará, surgen las diferencias y los argumentos encontrados debido a que cada grupo de poder pretende que se ponga en práctica aquella educación que, para su criterio, es la mejor. Es en esta situación donde juegan un papel importante los juicios de valor que promuevan una defensa de la educación que beneficie a todos (Fullat, 1989).

Los temas que pertenecen a la educación como realidad son la sociología, historia, psicología, economía, física, química, matemáticas, biología y otras materias técnicas. Los temas del proceso educativo que están enmarcados por el valor son la filosofía, ética, política, derecho, estética, entre otras. Queda diferenciada la dimensión fáctica del proceso educativo, de la dimensión axiológica, la cual es desiderativa y perfeccionadora (Casares, 1998; y Yurén, 2018). 


\section{El aspecto teleológico de la educación}

La educación requiere necesariamente, como punto de partida, la elaboración de un plan que facilite los criterios y brinde referencias de carácter, fundamentalmente, axiológicos. El punto de llegada de la educación es la materialización de los objetivos y finalidades propuestas en disposiciones y comportamientos comprometidos con los valores ofrecidos (Biesta, 2012).

De esta manera, por una parte, es necesario que la educación sea la que permita a cada hombre elaborar o desarrollar una habilidad para poder vivenciar determinados valores, estos deben ir en orden ascendente desde lo más individual a los sociales y altruistas como los más valiosos; y, por otra parte, es también tarea de la educación fomentar en el hombre el desarrollo de determinados modos de comportamiento que surjan de los valores adquiridos, es decir que aprenda a culturar los valores (Caride, 2017).

Si los fines propuestos son valiosos, la calidad de la educación dependerá de la coherencia entre los objetivos fijados y los alcanzados. Este patrón de proyecto, proceso y resultado hace referencia a lo axiológico y a lo normativo del avance del acto educativo, mediante el cual la persona se compromete con los valores en su profunda mismidad (Pereira, 2016). Es decir que, mientras más valiosos sean los estados finales, menos aleatorio será el comportamiento del hombre, a medida que se acerca a los patrones de valor y se desarrolla su acción educativa y autoeducativa. La educación se concreta en la articulación del comportamiento, de ahí la importancia de la pedagogía en la formación de las estructuras básicas hacia donde se orientará la acción educadora (Didier, 2017; Escalante, 2018; Galvis, López y Velásquez, 2018).

Además, el planteo de una educación integral de calidad implica que el modelo axiológico propuesto en el diseño curricular contenga el mayor número posible de valores a los que debe aspirar el hombre en cuanto tal y como miembro de una sociedad. De lo contrario, la educación se limita a la transmisión de conocimientos, perdiendo de vista el pleno desarrollo del hombre (Martínez, 2019). Es decir, es imprescindible para una educación integral lograr que la persona sea valiosa por sí misma y como miembro de la sociedad.

Desde una postura objetivista, se promueven los fines perseguidos por la educación de tal manera que el sujeto sienta la necesidad de alcanzar dichos valores. En consecuencia, la propuesta axiológica educativa debe tender a una formación integral de la persona, donde los valores sean 
percibidos como un fin en sí mismos (Álvarez, 2006). A fin de ilustrar esta reflexión, José Álvarez (2006) trae a colación las siguientes palabras de Fermoso:

(...) los fines educativos son fijados de acuerdo con una escala de valores aceptada, porque ésta es la que da importancia o no a los propósitos (...) Los fines educativos son entonces ideales, metas cargadas de atractivo que hacen desaparecer la indiferencia. (Fermoso, 1982; cit. en Álvarez, 2006, p. 122)

Los valores se presentan al hombre ordenados jerárquicamente en un modelo axiológico y éste debe elegirlos sin ningún tipo de coacción; debe preferirlos por lo que son y, si bien todas las cosas valen, no todas valen de la misma forma para todos, la fuerza en el orden jerárquico depende de la conjunción del sujeto, objeto y circunstancia. Todo ello establece una jerarquía personal desde donde el sujeto vive y toma sus decisiones, incluso, hasta las inconscientes. Sin embargo, la decisión que toma es fruto de un acto libre, si no hay libertad, la persona no ha asumido realmente los valores (Expósito, Marsollier y Difabio, 2018).

Es importante considerar que, en todos los sistemas educativos, la jerarquía axiológica obedece al arquetipo de hombre que se pretende formar desde un determinado modelo ideológico de base. En este sentido, no solo es imposible educar sin una referencia clara a un sistema de valores y normas; sino que ese sistema es anterior al planteo de los fines, en cuanto que son su fundamento (Expósito, 2018).

\section{Reflexiones finales}

La idea de una educación en valores radica en el principio de que su función es el perfeccionamiento del hombre, posibilitándole la creación y reformulación de los patrones planteados y sostenidos como valiosos (Gómez, 2017). Siempre la educación implica un proceso

orientado cuidadosamente hacia un tipo de persona considerado ideal. Éste es el patrón a seguir por la educación por ser inestimable en sí. Lo valioso hace deseable al modelo y su valor se analiza desde los aspectos considerados previamente en el desarrollo de este trabajo.

Si este planteo es estudiado desde una perspectiva subjetivista, el patrón se presenta deseable, por lo tanto, el compendio de valores que integran el modelo son las ideas, deseos e intereses del sujeto (Ruiz, 2017). De la misma manera sucede desde una perspectiva social de análisis, el individuo perteneciente a un determinado grupo valora aquellos patrones considerados 
importantes por sus pares. Esto no es un condicionante para que, en ciertos casos, surja una disociación o conflicto entre lo que el sujeto considera valioso respecto de lo que su grupo privilegia. Incluso puede suceder que, en determinadas situaciones concretas, lo que una institución o sujeto consideren valioso, sea diferente o contrapuesto a las valoraciones socialmente vigentes.

Por su parte, si el análisis tiene una raíz objetivista, se observan dos situaciones. Primero, los patrones están definidos por una serie de valores a priori, ajenos a la experiencia sensible. Segundo, la educación tendrá por objeto mostrarle al estudiante cuán valioso es el patrón para que éste lo conozca, lo estime y, como consecuencia, lo desee (Torres, Burbano y Narváez, 2019).

Finalmente, desde una visión integradora, los valores del modelo axiológico se configurarán teniendo en cuenta de igual modo al sujeto que al objeto y a la situación particular concreta.

Es necesario no perder de vista que cada sistema educativo está íntimamente ligado a una determinada estructura de valores aceptada por la ideología dominante y no contrapuesta con los anhelos sociales. Obviamente, antes de poner en marcha un proceso educativo, es necesario comprender desde qué modelo se está partiendo. Es ahí donde recién se puede vislumbrar qué se quiere educar debido a que no todos los valores valen lo mismo para todos los modelos ni para todas las ideologías. Es preciso tener bien en claro qué ideal de hombre se pretende alcanzar y cuál es su concepción educativa.

De esta manera, se hace evidente que es el arquetipo de hombre, propuesto desde una estructura axiológica en una determinada sociedad, el que orienta la vida de la persona organizando sus actividades y acciones; además, fundamenta y justifica que dichas acciones hayan sucedido en un determinado momento o que no sucedieran. El modelo axiológico se presenta como capaz de satisfacer todas las necesidades. También permite a cada persona generar una idea de sí misma y de cada sujeto con el que se relacione.

En consecuencia, cuando los actuales modelos ideológicos se ven interpelados por las grandes y tradicionales configuraciones axiológicas, se produce una crisis. A tal punto se llega en este juego de fricciones que hablar de valores individuales, morales y éticos se ha convertido en un verdadero dolor de cabeza. Incluso más, muchas veces se pretende escindirlos de los valores sociales bregando por imponer en la agenda sociopolítica aquellos temas denominados "tabú" 
como la educación sexual en las escuelas, la legalización del aborto, la eutanasia, la igualdad de género, la homosexualidad, las drogas, etc. Esto decanta en un proceso de reflexión por parte de cada ciudadano sobre el lugar que ocupan los valores en su vida personal y su comunidad como entorno favorable para el desarrollo pleno de ese hombre arquetípico.

\section{Referencias}

Álvarez, J. (2006). Los valores afectivos en la formación del profesorado. Cuestiones pedagógicas: Revista de ciencias de la educación, (18), 121-141. Recuperado de: https://dialnet.unirioja.es/servlet/articulo?codigo=3313942.

Aristóteles (1993). Ética Nicomáquea. Ética Eudemia. Traducido por P. Bonet, J. Madrid: Ed. Gredos.

Avilés, M. (2018). El lado oscuro en la educación: papel del símbolo en la formación de valores, espiritualidad e ideología. Cátedra, 1(1), 120-133. http://revistadigital.uce.edu.ec/index.php/CATEDRA/article/view/768

Berríos-Valenzuela, L., y Buxarrais-Estrada, M. R. (2013). Educación en valores: análisis sobre las expectativas y los valores de los adolescentes. Educación y educadores, 16(2), 244264. https://www.redalyc.org/pdf/834/83428615003.pdf

Biesta, G. J. (2012). Giving teaching back to education: Responding to the disappearance of the teacher. Phenomenology \& Practice, 6(2), 35-49. https://journals.library.ualberta.ca/pandpr/index.php/pandpr/article/view/19860

Brezinka, W. (2007). Educació en valors en una societat en crisi de valors. Revista Catalana de Pedagogia, 165-182. Recuperado de: http://revistes.iec.cat/index.php/RCP/article/view/3896.

Cantero, F. G., y García, D. R. (2012) Libro homenaje al profesor José Antonio Ibáñez-Martín Mellado. Biblioteca Online SL.

Caride, J. A. (2017). Educación social, derechos humanos y sostenibilidad en el desarrollo comunitario. Teoría de la Educación. 29(1), 245-272.

Casares, P. (1998). Los valores del profesorado en formación y su incidencia educativa (Tesis de doctorado) Universidad de Granada, España. 
Casillas, C. (2019). Currículum, ideología y capacidad crítica en la docencia universitaria. Revista Educación, 623-642.

Chancusi, A., \& Peralvo, C. (2016). La educación en valores asociada a la transversalidad en el currículo.Didasc@lia: Didáctica y Educación,7(6), 91-100. Recuperado de: http://refcale.uleam.edu.ec/index.php/didascalia/article/view/1192.

Didier, N. (2017). Los hijos de la democracia: rasgos, valores individuales, laborales y sociales en Chile. Revista de psicología (Santiago), 26(2), 50-65.

Escalante, A. (2018). Valores en la educación. Inventio. La génesis de la cultura universitaria en Morelos, 2(4), 31-40. Recuperado de: http://riaa.uaem.mx/handle/20.500.12055/353.

Expósito, C. (2018). De la reflexión ideológica a la realidad pedagógica.Apuntes Universitarios, 8(2). DOI: http://dx.doi.org/10.17162/au.v8i2.192.

Expósito, C., Marsollier, R., y Difabio, H. (2018). Los valores en educación para una educación sin valores. Dilemas Contemporáneos: Educación, Política y Valores, 5(2) 38.

Recuperado de:

https://www.dilemascontemporaneoseducacionpoliticayvalores.com/edici\%e2\%99\%80n -2013/ano-v-publicacion-no-2-enero-2018/.

Ferrández, A. y Sarramona, J. (1997) La educación. Constantes y problemática actual. Barcelona. CEAC.

Fullat, O. (1989). Educación. F. Altarejos y otros. Filosofía de la Educación hoy. Madrid, Dykinson.

Galvis, L., López-Díaz, L., y Velásquez, V. (2018). Patrones culturales de cuidado familiar al adulto mayor en condición de discapacidad y pobreza. Index de Enfermería, 27(3), 170174. Recuperado de: http://scielo.isciii.es/scielo.php?pid=S1132$12962018000200013 \&$ script=sci_arttext\&tlng=pt.

García Hoz, V. (1993). Introducción general a una pedagogía de la persona (Vol. 1). Ediciones Rialp.

García, L., Ruiz, M., y García, M. (2016). Claves para la educación: Actores, agentes y escenarios en la sociedad actual. Narcea Ediciones.

Gervilla, E. (2000). Un modelo axiológico de educación integral. Revista española de pedagogía, 58(215), 39-57. 
Gómez, E. L. (2017). Educación en valores. Revista Raites, 3(6) 69-87. Recuperado de: http://www.itcelaya.edu.mx/ojs/index.php/raites/issue/view/41.

Gracia, J., y Gozálvez, V. (2016). Justificación filosófica de la educación en valores éticos y cívicos en la educación formal. Análisis crítico de la LOMCE. Teoría de la Educación. Revista Interuniversitaria, 28(1), 83-103. doi:10.14201/teri.13108

Hall, B. P. (2006). Values shift: A guide to personal and organizational transformation. Estados Unidos: Wipf and Stock Publishers.

Hernández-Romero, G. (2020). El respeto: un valor que desafía a la educación de universitarios tabasqueños en la modernidad liquida. Apuntes Universitarios, 10(3), 131 - 145. https://doi.org/10.17162/au.v10i3.475

Kant, I (2009). Sobre Pedagogía. Traducido por O. Caeiro. Argentina: Editorial Universidad Nacional de Córdoba.

Ley $N^{\circ}$ 26206, de Educación Nacional. Boletín Oficial del Estado, 28. Argentina. 27 de diciembre de 2006.

Martínez, P. (2017). Tutoría en acción. Educatio Siglo XXI, 35(2 Jul-Oct), 11-20.

Martínez, V. (2019). Claves axiológicas y retos educativos en Iberoamérica. Revista Iberoamericana de Educación, 80(2), 105-127. DOI: https://doi.org/10.35362/rie8023316.

Pereira, G. (2016). Emancipación y educación. Ludus Vitalis, 15(28), 231-235. Recuperado de: http://www.ludus-vitalis.org/ojs/index.php/ludus/article/viewFile/379/369.

Ramírez, A., Hernández, J., y Viamontes, E. (2018). Consideraciones acerca de la formación de valores en la Educación Superior. Opuntia Brava,10(1), 194-206. DOI: https://doi.org/10.35195/ob.v10i1.67

Ruiz, A. (2017). Importancia de los valores humanos en la educación. Daena: International Journal of Good Conscience, 17. Recuperado de: http://www.spentamexico.org/v12n3/A21.12(3)345-356.pdf

Santos, E. (2016). Los valores como resultado de la relación Dios-hombre y conducta empresarial. Apuntes universitarios, 6(2). DOI: https://doi.org/10.17162/au.v6i2.216

Sanvisens, A. (1984). Educación, pedagogía y ciencias de la educación. Sanvisens, A. y otros Introducción a la Pedagogía. Barcelona: Barcanova. 
Sauter, G. O., y Rodríguez, J. M. S. (2005). Los manuales escolares como fuente para la historia de la educación en América Latina. Editorial UNED.

Torres, Á., Burbano, E., \& Narváez, A. (2019). El sentido de la educación en valores: un camino para la construcción de la paz. Huellas Revista. 1(11) 63-78. Recuperado de: https://revistas.udenar.edu.co/index.php/rhuellas/issue/view/436.

Torres, M. (2002). Los valores, aplicación en la educación y conceptualización histórica. Doctoral dissertation, UPN-97. México. Recuperado de: http://hdl.handle.net/123456789/829.

Touriñán López, J. M. (2005). Educación en Valores, Educación Intercultural y Formación para la Convivencia. Netbiblo.

UNESCO (1974) Recomendación sobre la educación para la comprensión, la cooperación y la paz internacionales y la educación relativa a los derechos humanos y las libertades fundamentales Aprobada por la Conferencia General de la Organización de las Naciones Unidas para la Educación, la Ciencia y la Cultura, en su 18. a reunión el 19 de noviembre de 1974.

Yurén, M. T. (2018). Adolfo Sánchez Vázquez, una veta para la educación valora. Inventio, la génesis de la cultura universitaria en Morelos,2(3), 61-70. Recuperado de: http://inventio.uaem.mx/index.php/inventio/article/view/175. 